Un gène de maladie d'Alzhcimer familiale localisé sur le chromosome 14. Les formes familiales de la maladie d'Alzheimer sont génétiquement hétérogènes. On connaît un locus sur le chromosome 21 , et quelques rares cas peuvent être attribués à des mutations sur la protéinc APP (précurseur de la protéine amyloïde) $\left(\mathrm{m} / \mathrm{s} n^{\circ} 1\right.$, vol. 8, p. $81 \mathrm{et}$ $n^{\circ} 8$, vol. 8, p. 866). Un autre locus, moins bien défini, pourrait se trouver sur le bras long du chromosome 19 [1]. une équipe américaine (13 auteurs) a étudié [2] des familles ayant présenté un début " précoce" (avant 60 ans), avec au moins trois sujets affectés sur au moins deux générations. Une liaison étroite (lod score dépassant 9) a été mise en évidence avec deux sondes, D14S43 et D14S53, correspondant à la bande q24.3 du chromosome 14 ; cette localisation ne s'applique toutefois pas aux descendants des Allemands de la
Volga, qui échappent encore à toute précision. Ces résultats sont confirmés par une étude toute récente portant sur neuf familles $[3,4]$. Parmi les gènes candidats possibles situés en $14 \mathrm{q} 24$, les auteurs [2] citent $c$-fos (qui pourrait être impliqué dans la régulation du gène APP) et HSPA2 (protéine du choc thermique à $70 \mathrm{kDa}$ ), qui est une molécule chaperonne intervenant dans l'assemblée des protéines.

[1. Pericak-Vance MA, et al. Am J Hum Genet 1991 ; 48 : 1034-50.]

[2. Schellenberg GD, et al. Science $1992 ; 258$ : 668-75.|

[3. Mullan M. Br Med J 1992 ; 305 : 1108$.

[4. Mullan M, et al. Nature Genet 1993 (sous presse).]

Les pseudo-maladics de TaySachs. Le déficit en $\beta$-hexosaminidase A provoque la redoutable maladie de
Tay-Sachs ( $\mathrm{m} / \mathrm{s} n^{\circ} 8$, vol. 9, p. 797). On connaît cependant des sujets normaux qui présentent un déficit lorsqu'on mesure l'activité de l'enzyme in vitro. Ils sont appelés des pseudo-déficits. L'équipe canadienne de Gravel, avec des chercheurs des États-Unis (13 auteurs), a montré qu'une mutation Arg $247 \rightarrow$ Trp en était responsable. Dans les familles à risque pour la maladie de Tay-Sachs, cette mutation sur un allèle accompagne une mutation Tay-Sachs vraie sur l'autre allèle. Elle a été trouvée chez 32 \% (20/62) porteurs de TaySachs non Juifs ashkénazes et chez aucun des 36 porteurs ashkénazes examinés. Étant donné cette fréquence, le criblage pour la mutation paraît essentiel afin d'éviter des interruptions de grossesse chez des enfants cliniquement sains.

[Triggs-Raine BL, et al. Am J Hum Genet 1992 ; 51 : 793-801.]

\title{
L'ADNC DU RÉCEPTEUR $\delta$ DES ENKÉPHALINES EST ENFIN CLONÉ !
}

La morphine, alcaloide extrait de l'opium, est connue depuis plusieurs dizaines d'années. C'est un analgésique puissant couramment utilisé en médecine, et l'étude de son mode $d^{\prime}$ action sur le système nerveux a permis de comprendre qu'elle agit sur des récepteurs spécifiques que l'on appelle aujourd'hui les récepteurs opiacés. Ces récepteurs membranaires du système nerveux sont impliqués dans la réponse de l'organisme à différents types de stress et en particulier à la douleur. Ils jouent un rôle également dans des mécanismes centraux tels que le contrôle de l'affectivité, les mécanismes d'attention et la capacité d'apprentissage. L'activation d'un récepteur opiacé produit typiquement un effet inhibiteur sur la cellule : ralentissement de la propogation de l'influx nerveux et inhibition de la libération des neuromédiateurs. Les études pharmacologiques leur capacité à lier les différents ligands opioides et par leur distribution dans l'organisme [1]. Les sous-types $\mu$, $\delta$ et $\kappa$ ont une affinité élevée pour, respectivement, la morphine, les enképhalines et les dynorphines. Ce que I'on sait aujourd'hui sur les récepteurs opioides est déduit à partir d'études basées sur l'utilisation de ligands opioides - synthétiques ou naturels - en présence de tissus contenant une population hétérogène de récepteurs.

La progression des connaissances dans ce domaine est actuellement limitée par l'absence de caractérisation moléculaire de ces récepteurs. Les nombreuses tentatives effectuées depuis plusieurs années ont échoué, probablement en raison de la rareté et de la nature très instable de ces molécules. L'équipe de Brigitte Kieffer, localisée à l'École Supérieure de Biotechnologie de Strasbourg (ESBS) a isolé un ADN complémentaire $(A D N c)$ codant pour le récepteur $\delta$, gråce à une méthode récente de cloprimé dans une cellule de mammifère hétérologue (cellule COS) produit un récepteur recombinant qui présente toutes les caractéristiques pharmacologiques du récepteur natif. En effet, il fixe le ${ }^{3} H D T L E T$, un agoniste delta, avec la même affinité que le récepteur d'origine dans les cellules NG108-15, il est stéréoselectif et fixe préférentiellement les ligands $\delta$ par rapport aux ligands $\mu$ et $\kappa$.

L'analyse de la séquence de l'ADNc codant pour ce récepteur et par ont démontré l'existence de trois sous-types de récepteurs, différant par nage par expression dans des cellules de mammifères [2]. L'ADNc surex-

déduction de la séquence en acides aminés, montre sans ambiguité que ce récepteur fait partie de la famille des récepteurs couplés aux protéines $G$ (protéines liant le GTP] [3], dont un exemple classique est le récepteur adrénergique. Cela est en accord avec des expériences biochimiques antérieures montrant que la réponse opioide est liée à l'activation de protéines $G$ de type Gi, Go ou Gk. Le profil d'hydrophobicité est en accord avec une topologie à sept domaines transmembranaires, structure com munément admise pour cette famille de récepteurs. Cette séquence est globalement peu homologue à celle des autres récepteurs de la même famille. Les récepteurs les plus similaires sont ceux de la somatostatine et du N-formylpeptide [4] avec qui elle présente $30 \%$ d'identité. Enfin, cette séquence occupe une place particulière parmi le groupe des récepteurs couplés aux protéines $G$, que l'on divise généralement en deux sous. groupes bien distincts, celui des récepteurs fixant les catécholamines et celui de ceux fixant les ligands peptidiques: le récepteur opioide delta contient un résidu aspartique en position 128 qui est caractéristique des récepteurs à catécholamine, mais contient également une troisième bou cle cytoplasmique très courte qui est typique des récepteurs à peptides. Grâce à ce premier clone d'ADNc codant pour le récepteur $\delta$, on peut espérer cloner les autres sous-types de récepteurs opiacés par homologie, et donc réaliser la caractérisation moléculaire et l'étude fonctionnelle des différents sous-types de récepteurs opiacés. Une meilleure compréhension des événements moléculaires relayés par ces récepteurs devra permettre alors le développement de nouveaux opioides à action plus spécifique et donc de disposer d'analgésiques puissants dépourvus d'effets secondaires ainsi que de drogues de substitution efficaces pour le traitement de la toxicomanie.

11. Simonds WF. Endocrinol Rev 1988; $9: 200-12$.

12. Kieffer B, et al. Proc Natl Acad Sci USA 1992: 89 (sous pressel.)

13. Bockaert J. Curr Op Neurobiol 1991; 1 : 32-42.]

14. Morel F, et al. médecine/sciences $1992 ; 8: 912-20$ J.

Brigitte Kieffer Claire Gavériaux-Ruff Katia Befor 